\title{
STONE-ČECH COMPACTIFICATIONS OF PRODUCTS $\left({ }^{1}\right)$
}

\author{
BY \\ IRVING GLICKSBERG
}

1. Introduction. As is well known from the work of Tychonoff [10], Stone [8], and Čech [1], every completely regular space $X$ can be imbedded as a dense subspace of a compact Hausdorff space $\beta(X)$ in such a way that continuous (real valued and bounded) functions on $X$ extend continuously to $\beta(X)$; indeed the resulting compactification of $X$, the Stone-Čech compactification, is uniquely determined by just these properties. For a set of completely regular spaces, the naturally induced imbedding of their product $P X_{\alpha}$ as a dense subspace of $P \beta\left(X_{\alpha}\right)$ yields a compactification of their product, and the question arises as to when one can identify $\left({ }^{2}\right)$ this with the Stone-Čech compactification. The main purpose of this paper is to show that aside from a trivial case, this identification is possible if and only if $P X_{\alpha}$ is pseudo-compact $\left(^{3}\right)$ [5], i.e., if and only if every real valued continuous function on it is bounded, or, equivalently, every bounded continuous function assumes its bounds $\left({ }^{4}\right)$. A side result of the investigation is the fact that every product of uncountably many compact spaces, each having at least two points, is the Stone-Cech compactification of certain proper subspaces, yielding a fairly accessible body of nontrivial Stone-Čech compactifications. Finally we shall give several conditions sufficient to insure that a product of pseudo-compact spaces be pseudo-compact, and briefly discuss a related question.

2. Pseudo-compact spaces. Several facts concerning pseudo-compact spaces will be used repeatedly in the following pages, and we shall collect them here. One of our main tools is a characteristic property, that Ascoli's theorem holds [3, Theorem 2], that is, every bounded equicontinuous set of functions on a pseudo-compact space $X$ has compact closure in the Banach

Received by the editors November 2, 1956 and, in revised form, May 6, 1957.

(1) Some of the results of this paper (essentially the necessity in Theorem 1, and Theorem 3) were originally included in a separate note submitted to the Proc. Amer. Math. Soc. in 1955, and were obtained while the writer was at The RAND Corporation. The same part of Theorem 1 has been obtained by M. Henriksen and J. R. Isbell [4] who also obtained some results in the converse direction. The writer would like to express his thanks to Professors Henriksen and Isbell for allowing him to read their manuscript.

(2) We shall simply write $\beta\left(P X_{\alpha}\right)=P \beta\left(X_{\alpha}\right)$ to express this identity (rather than the identity of the compact spaces involved (cf. §6)); when the meaning is clear we shall speak of $\beta(X)$ as the compactification. It will be convenient to always consider $X$ as a subspace of $\beta(X)$, and thus $P X_{\alpha}$ as a subspace of $\boldsymbol{P} \beta\left(X_{\alpha}\right)$.

(3) It should perhaps be noted that this corrects an erroneous assertion made by Hewitt [5, Theorem 14].

(4) In addition pseudo-compactness requires complete regularity. 
space $C(X)$ of all bounded real valued continuous functions on $X$. A direct corollary is the fact that, for a bounded set of functions on such a space, equicontinuity is equivalent to equicontinuity of each countable subset, since conditional compactness and conditional countable compactness coincide in $C(X)$.

Another characteristic property of these spaces which will prove useful is purely topological in form [3, Theorem 2]: every sequence of nonvoid, pairwise disjoint open sets has a cluster point (which by definition has the property that each of its neighborhoods meets infinitely many elements of the sequence). Of course such a cluster point prevents the sequence from forming a locally finite collection of open sets. On the other hand, given an infinite locally finite collection $C$ of open sets, on any space, we can produce a sequence of the type described with no cluster point: we simply choose an open neighborhood $N_{1}$ contained in one element of $C$ and meeting only finitely many; deleting these from $C$ to form $C_{1}$ we obtain a similar neighborhood $N_{2}$ for $C_{1}$, and continue. Clearly the sequence $\left\{N_{i}\right\}$ we obtain, being locally finite, has no cluster point, and is easily seen to satisfy our other requirements. Consequently, if a space is pseudo-compact then every locally finite collection of open sets is finite. The converse of this assertion is obvious, so we have another characteristic property, which in turn yields the following strengthened form of our original topological condition: every sequence of nonvoid open sets has a cluster point if and only if the space is pseudo-compact. We need only verify that a pseudo-compact space has the required property. But a sequence of nonvoid open sets yields a collection of sets which is locally finite or not; in the first case the collection is finite so that some point lies in infinitely many sets of the sequence, and is thus a cluster point. In the second case some point prohibits local finiteness, and clearly this point is the required cluster point.

Pseudo-compactness is also reflected in the relationship between $\beta(X)$ and its subspace $X: X$ is pseudo-compact if and only if there is no nonvoid closed $G_{\delta}$ in $\beta(X) \backslash X$. For, given a nonvoid closed $G_{\delta}$ in $\beta(X) \backslash X$, we can produce by Urysohn's lemma a continuous function on $\beta(X)$ that does not assume its maximum value on the dense subspace $X$; conversely, given a continuous bounded function on $X$ which does not assume its least upper bound, the set where the continuous extension of this function to $\beta(X)$ assumes its maximum is a nonvoid closed $G_{\delta}$ in $\beta(X) \backslash X$.

If a product $P X_{\alpha}$ is pseudo-compact, clearly each factor space and each partial product is also pseudo-compact, since continuous functions on these spaces may be considered as continuous functions on the full product. Further, pseudo-compactness of the product is equivalent to pseudo-compactness of every countable partial product $P_{i=1}^{\infty} X_{\alpha_{i}}$. For, since it suffices to show that every sequence of (nonvoid) canonical neighborhoods in $P X_{\alpha}$ has a cluster 
point, and each neighborhood places restrictions on only finitely many coordinates, only countably many coordinates are involved, say those corresponding to $\alpha_{1}, \alpha_{2}, \ldots$. Choosing any cluster point of the projection of our sequence into $P X_{\alpha_{i}}$, we can (by any arbitrary choice of all other coordinates) clearly extend this point to a cluster point of the original sequence.

Notation. We shall allow $x$ to represent a generic element of $\beta(X)$ rather than of $X$. For $f \in C(X), f^{*}$ will denote its unique continuous extension to $\beta(X), f \mid F$ its restriction to a subset $F$ of $X$. For such a subset $F^{-}$and $F^{\prime}$ will denote closure and complement, respectively, in $X$. Where no confusion can arise we shall refer to $\beta(X) \backslash X$ as the set of ideal points of $\beta(X)$. All spaces will be assumed completely regular, and all functions real valued.

3. The main result. If, for some $\alpha_{0}, P_{\alpha \neq \alpha_{0}} X_{\alpha}$ forms a finite set, then every $f \in C\left(P X_{\alpha}\right)$ clearly has a continuous extension to $P \beta\left(X_{\alpha}\right)$, and we may write $\beta\left(P X_{\alpha}\right)=P \beta\left(X_{\alpha}\right)$. This is precisely the trivial case mentioned in the introduction.

THEOREM 1. Let $\left\{X_{\alpha}\right\}$ be a set of completely regular spaces, and suppose the set $\boldsymbol{P}_{\alpha \neq \alpha_{0}} X_{\alpha}$ is infinite for every $\alpha_{0}$. Then a necessary and sufficient condition that $\beta\left(P X_{\alpha}\right)=P \beta\left(X_{\alpha}\right)$ is that $P X_{\alpha}$ be pseudo-compact.

Proof of the necessity. A moment's reflection shows that the property $\beta\left(P X_{\alpha}\right)=P \beta\left(X_{\alpha}\right)$ is inherited by all partial products $\left(^{5}\right)$; consequently, for any subset $B$ of our index set, we may evidently write

$$
\begin{aligned}
\beta\left(\underset{\alpha \in B}{P} X_{\alpha} \times \underset{\alpha \notin B}{P} X_{\alpha}\right) & =P \beta\left(X_{\alpha}\right)=\underset{\alpha \in B}{P} \beta\left(X_{\alpha}\right) \times \underset{\alpha \ddagger_{B}}{P} \beta\left(X_{\alpha}\right) \\
& =\beta\left(\underset{\alpha \in B}{P} X_{\alpha}\right) \times \beta\left(\underset{\alpha \oplus_{B}}{P} X_{\alpha}\right) .
\end{aligned}
$$

Thus it will suffice to prove that if $X$ and $Y$ are infinite spaces such that $\beta(X \times Y)=\beta(X) \times \beta(Y)$, then $X \times Y$ is pseudo-compact. We shall first show that each of the spaces $X$ and $Y$ is pseudo-compact. In showing $Y$ is pseudocompact, we can assume $X$ is compact. For if $f \in C(\beta(X) \times Y)$, then $(f \mid X \times Y)^{*}$ * clearly must coincide with $f$ on $\beta(X) \times Y$, and thus continuously extends $f$ to $\beta(X) \times \beta(Y)$; thus $\beta(\beta(X) \times Y)=\beta(X) \times \beta(Y)$, and we may replace $X$ by $\beta(X)$.

Suppose then that $Y$ is not pseudo-compact and that $X$ is compact. Then there is a $g$ in $C(Y)$ which never vanishes on $Y$ but has zero as its greatest lower bound, and hence has a sequence of values $g\left(y_{n}\right) \rightarrow 0$. Since $X$ is infinite,

(5) For we may identify the partial product $P_{\alpha \in B} X_{\alpha}$ with the subspace $P_{\alpha \in B} X_{\alpha} \times\{x\}$ of $\boldsymbol{P} \beta\left(X_{\alpha}\right)$ (where $x \in \boldsymbol{P}_{\alpha \notin B} X_{\alpha}$ ), and similarly $\boldsymbol{P}_{\alpha \in B} \beta\left(X_{\alpha}\right)$ with $\boldsymbol{P}_{\alpha \in B} \beta\left(X_{\alpha}\right) \times\{x\}$; since $f \in C\left(\boldsymbol{P}_{\alpha \in B} X_{\alpha} \times\{x\}\right)$ has an obvious continuous extension to $\boldsymbol{P} X_{\alpha}$, it has one to $\boldsymbol{P} \beta\left(X_{\alpha}\right)$, hence to $P_{\alpha \in B} \beta\left(X_{\alpha}\right) \times\{x\}$. 
there is an $f \in C(X)$ having infinitely many values $\left({ }^{6}\right)$, and we may assume that a sequence of these, $\left\{f\left(x_{n}\right)\right\}$, strictly decreases to zero. By linear interpolation, we can construct a non-negative, continuous, bounded function $h$ on the reals for which $h\left(f\left(x_{n}\right)\right)=g\left(y_{n}\right)$, and setting

$$
\phi(x, y)=2 h(f(x)) g(y) /\left(h(f(x))^{2}+g(y)^{2}\right),
$$

we obtain an element $\phi$ of $C(X \times Y)$ : for $g$ never vanishes and $0 \leqq \phi \leqq 1$. But consider $\phi^{*}$. If $(x, y)$ is a cluster point of the sequence $\left\{\left(x_{n}, y_{n}\right)\right\}$ in the compact space $X \times \beta(Y)$, then $\phi^{*}(x, y)=1$ since $\phi^{*}\left(x_{n}, y_{n}\right)=\phi\left(x_{n}, y_{n}\right)=1$. On the other hand, $x$ is a cluster point of $\left\{x_{n}\right\}$ and, since $\phi^{*}\left(x_{n}, y^{\prime}\right)=\phi\left(x_{n}, y^{\prime}\right)$ $\rightarrow 0$ for each $y^{\prime}$ in $Y$, we have $\phi^{*}\left(x, y^{\prime}\right)=0$ for each $y^{\prime}$ in $Y$. Since $Y$ is dense in $\beta(Y)$, it follows that $\phi^{*}(x, y)=0$, which is the desired contradiction.

Returning to the general case, we see that both $X$ and $Y$ must be pseudocompact. In order to see that this is also the case for $X \times Y$, we need only prove that any closed $G_{\delta}$ in the set of ideal points of $\beta(X \times Y)$ must be void (cf. §2). But if $F$ is such a subset of the set of ideal points of $\beta(X \times Y)$, then, for each $x$ in $X, F \cap(\{x\} \times \beta(Y))$ is a closed $G_{\delta}$ in the set of ideal points of $\beta(\{x\} \times Y)=\{x\} \times \beta(Y)$, and thus is void since $\{x\} \times Y$ is pseudo-compact. Hence $F \cap(X \times \beta(Y))$ is void and $F \subset(\beta(X) \backslash X) \times \beta(Y)$. But now for every $y$ in $\beta(Y), F \cap(\beta(X) \times\{y\})$ is a closed $G_{\delta}$ in the set of ideal points of $\beta(X \times\{y\})$ $=\beta(X) \times\{y\}$, hence void, so that $F=F \cap(\beta(X) \times \beta(Y))$ must be void, and $X \times Y$ must be pseudo-compact.

Our proof of the sufficiency is based on the possibility of coordinate-wise extension of an element of $C\left(P X_{\alpha}\right)$, which results, in the case of finitely many factors, from the following lemmas.

Lemma 1. If $X \times Y$ is pseudo-compact and $f \in C(X \times Y)$, then the family $\{f(x, \cdot): x \in X\}$ is equicontinuous on $Y$. Consequently the mapping $y \rightarrow f(\cdot, y)$ of $Y$ into $C(X)$ is continuous.

Proof. Since the second statement of the lemma is an obvious consequence of the first, and since $Y$ is pseudo-compact, it is sufficient to prove that any sequence $\left\{f\left(x_{n}^{\prime}, \cdot\right)\right\}$ is equicontinuous on $Y$ (cf. §2). Suppose not. Then for some $y_{0}$ in $Y$ and $\epsilon>0$, no neighborhood $W$ of $y_{0}$ satisfies the condition

$$
\left|f\left(x_{n}^{\prime}, y\right)-f\left(x_{n}^{\prime}, y_{0}\right)\right|<\epsilon \quad \text { for } y \in W,
$$

for all $n$. We now select a subsequence $\left\{x_{n}\right\}$ of $\left\{x_{n}^{\prime}\right\}$ and open neighborhoods $V_{n}$ of $x_{n}, W_{n}$ of $y_{0}$ as follows. Let $W_{1}=Y$ and $x_{1}$ be the first $x_{n}^{\prime}$ for which (*) fails for $W=W_{1}$. We choose a neighborhood $V_{1} \times W_{2}$ of $\left(x_{1}, y_{0}\right)$ on which $f$ varies by $<1$; having chosen $x_{1}, \cdots, x_{k}, V_{1}, \cdots, V_{k}, W_{1}, \cdots, W_{k+1}$ we

${ }^{6}$ ) The following argument is due to the referee. If continuous functions with only finitely many values suffice to separate every pair of points, then it is easy to see that $X$ contains a strictly decreasing sequence of open and closed subsets. Taking $f_{n}$ as the characteristic function of the $n$th subset, then $f=\sum 2^{-n} f_{n}$ is continuous and assumes infinitely many values. 
select $x_{k+1}$ as the first $x_{n}^{\prime}$ for which (*) fails for $W=W_{k+1}$, and select an open neighborhood $V_{k+1} \times W_{k+2}$ of $\left(x_{k+1}, y_{0}\right)$ on which $f$ varies by $<1 /(k+1)$, with $W_{\boldsymbol{k}+2}^{-} \subset W_{k+1}$.

From our choice of $x_{n}$ we have a $y_{n}$ in $W_{n}$ for which $\left|f\left(x_{n}, y_{n}\right)-f\left(x_{n}, y_{0}\right)\right|$ $\geqq \epsilon$; consequently we may choose an open neighborhood $\tilde{V}_{n} \times \tilde{W}_{n}$ of $\left(x_{n}, y_{n}\right)$ lying within $V_{n} \times W_{n}$ which yields $\left|f(x, y)-f\left(x, y_{0}\right)\right|>\epsilon / 2$ for $(x, y) \in \tilde{V}_{n} \times \widetilde{W}_{n}$. But by one of our topological conditions for pseudo-compactness, $\left\{\widetilde{V}_{n} \times \widetilde{W}_{n}\right\}$ has a cluster point $(\bar{x}, \bar{y})$ in $X \times Y$, so that $\left|f(\bar{x}, \bar{y})-f\left(\bar{x}, y_{0}\right)\right| \geqq \epsilon / 2$ by continuity. On the other hand, as a cluster point of $\left\{\widetilde{W}_{n}\right\}, \bar{y} \in \bigcap_{j=1}^{\infty} W_{j}$; for $\bar{y} \notin W_{k}$ implies $\bar{y} \notin W_{k+1}^{-}$so that $W_{k+1}^{-\prime}$ is a neighborhood of $\bar{y}$ meeting only finitely many $\tilde{W}_{n}$. Thus for $x \in \tilde{V}_{n} \subset V_{n},\left|f(x, \bar{y})-f\left(x, y_{0}\right)\right|<1 / n$ since both $(x, \bar{y})$ and $\left(x, y_{0}\right)$ lie in $V_{n} \times W_{n+1}$. Since $\bar{x}$ is a cluster point of $\left\{\tilde{V}_{n}\right\}, 0$ $=\left|f(\bar{x}, \bar{y})-f\left(\bar{x}, y_{0}\right)\right| \geqq \epsilon / 2$, a contradiction establishing equicontinuity, and completing the proof.

Lemma 2. Let $X$ and $Y$ be completely regular and $f \in C(X \times Y)$. If the mapping $y \rightarrow f(\cdot, y)$ of $Y$ into $C(X)$ is continuous, then $f$ has a continuous extension to $\beta(X) \times Y$.

Proof. In view of the natural isomorphism of $C(X)$ and $C(\beta(X))$, continuity of the mapping $y \rightarrow f(\cdot, y)$ implies continuity of the mapping $y \rightarrow \bar{f}(\cdot, y)$ of $Y$ into $C(\beta(X))$, where $\bar{f}(\cdot, y)$ is the unique continuous extension of $f(\cdot, y)$ to $\beta(X)$. But as a function on $\beta(X) \times Y, \bar{f}$ is continuous. For given $\left(x_{0}, y_{0}\right)$ $\in \beta(X) \times Y$ and $\epsilon>0$, we have a neighborhood $V$ of $x_{0}$ satisfying $\mid \bar{f}\left(x, y_{0}\right)$ $-\bar{f}\left(x_{0}, y_{0}\right) \mid<\epsilon$ for $x \in V$, since $\bar{f}\left(\cdot, y_{0}\right) \in C(\beta(X))$; since $y \rightarrow \bar{f}(\cdot, y)$ is continuous, $y_{0}$ has a neighborhood $W$ satisfying $\left|\bar{f}(x, y)-\bar{f}\left(x, y_{0}\right)\right|<\epsilon$ for $y \in W$ and all $x$ in $\beta(X)$. Thus for $(x, y) \in V \times W,\left|\bar{f}(x, y)-\bar{f}\left(x_{0}, y_{0}\right)\right| \leqq\left|\bar{f}(x, y)-\bar{f}\left(x, y_{0}\right)\right|$ $+\left|\bar{f}\left(x, y_{0}\right)-\bar{f}\left(x_{0}, y_{0}\right)\right|<2 \epsilon$, which establishes continuity and completes the proof of Lemma 2.

Proof of the sufficiency in Theorem 1. If $X \times Y$ is pseudo-compact, then by Lemmas 1 and 2 we may extend $f \in C(X \times Y)$ continuously to $\beta(X) \times Y$. Now any space containing a dense pseudo-compact subspace is pseudocompact (since any continuous function on the space, being bounded on the dense subspace, is bounded); thus $\beta(X) \times Y$ is pseudo-compact and applying the lemmas once more we obtain a continuous extension to $\beta(X) \times \beta(Y)$, and the proof is complete for the case of two factor spaces. Since the result now follows immediately for a finite set of factors, we may turn to the infinite case.

Suppose that $P X_{\alpha}$ is pseudo-compact and $f \in C\left(P X_{\alpha}\right)$. Then for every $\epsilon>0$, there is a finite set $\alpha_{1}, \cdots, \alpha_{n}$ of indices for which $x, y \in P X_{\alpha}, x_{\alpha_{i}}=y_{\alpha_{i}}$, $i=1, \cdots, n$, imply $|f(x)-f(y)|<\epsilon$. Suppose not, and let $F_{0}$ be any finite set of coordinate indices. Then we have points $x^{1}$ and $y^{1}$, agreeing in those coordinates with $\alpha \in F_{0}$, with $\left|f\left(x^{1}\right)-f\left(y^{1}\right)\right| \geqq \epsilon$. By continuity and the form of neighborhoods in the product we may clearly assume, if we replace $\epsilon$ by 
$\epsilon / 2$, that $x^{1}$ and $y^{1}$ differ in only finitely many coordinates, say those corresponding to $\alpha \in F_{1}$. Considering the finite set $F_{0} \cup F_{1}$, we can arrive in the same fashion at two points $x^{2}$ and $y^{2}$ with $\left|f\left(x^{2}\right)-f\left(y^{2}\right)\right| \geqq \epsilon / 2$, agreeing in those coordinates with $\alpha \in F_{0} \cup F_{1}$ and differing in just those coordinates with $\alpha \in F_{2}, F_{2}$ finite. Continuing, we obtain sequences $\left\{x^{i}\right\},\left\{y^{i}\right\},\left\{F_{i}\right\}$ for which $\left|f\left(x^{i}\right)-f\left(y^{i}\right)\right| \geqq \epsilon / 2, x^{i}$ and $y^{i}$ differ in only those coordinates with $\alpha \in F_{i}$, and the $F_{i}$ are finite and pairwise disjoint.

Now, by continuity, we can enlarge each $x^{i}$ to a canonical open neighborhood $U^{i}$ and each $y^{i}$ to a canonical open neighborhood $V^{i}$ so that $|f(x)-f(y)|$ $>\epsilon / 4$ for $x \in U^{i}, y \in V^{i}$. Indeed we can clearly insist that $U^{i}$ and $V^{i}$ agree in all their components except those in which $x^{i}$ and $y^{i}$ disagree, i.e., for $\alpha \in F_{i}$. But $\left\{U^{i}\right\}$ has a cluster point $\bar{x}$, and if $W$ is a canonical open neighborhood of $\bar{x}$, placing restrictions on only those coordinates corresponding to $\alpha_{1}, \cdots$, $\alpha_{n}$, then we have $W$ meeting $U^{i}$ if and only if $W$ meets $V^{i}$ for $i$ large enough to yield $\left\{\alpha_{1}, \cdots, \alpha_{n}\right\} \cap \bigcup_{j=1}^{\infty} F_{j} \subset \bigcup_{j=1}^{i-1} F_{j}$ (since $U^{i}$ and $V^{i}$ then have the same $\alpha_{1}$ st, $\cdots, \alpha_{n}$ th components). Consequently each neighborhood of $\bar{x}$ contains a pair of points satisfying $|f(x)-f(y)|>\epsilon / 4$, contradicting continuity, and establishing our assertion.

Now let us consider $f$ as defined on the subspace $P X_{\alpha}$ of $P \beta\left(X_{\alpha}\right)$. If $f$ has no continuous extension to $P \beta\left(X_{\alpha}\right)$, we have an element $x^{0}$ of this space for which $\lim \sup _{x \rightarrow x^{0}} f(x)-\lim \inf _{x \rightarrow x^{0}} f(x)=a>0$, where $x$ is taken from the dense subspace $P X_{\alpha}$. Let $0<3 \epsilon<a$ and $\alpha_{1}, \cdots, \alpha_{n}$ be the finite set of indices obtained for this $\epsilon$ in the previous paragraph. For brevity let us now write $X=P_{i=1}^{n} X_{\alpha_{i}}, Y=P_{\alpha \neq \alpha_{1}, \cdots, \alpha_{n}} X_{\alpha}$ and the values of $f$ as for a function on $X \times Y$.

By Lemmas 1 and $2, f$ has a continuous extension $\bar{f}$ to $\beta(X) \times Y$ $=P_{i=1}^{n} \beta\left(X_{\alpha_{i}}\right) \times Y$, a subspace of $P \beta\left(X_{\alpha}\right)$. Moreover since $\beta(X) \times Y$ is pseudocompact, by Lemma $1\{\bar{f}(\cdot, y)\}_{y \in Y}$ is equicontinuous on $\beta(X)$. In particular, this is the case at $\left(x_{\alpha_{1}}^{0}, \cdots, x_{\alpha_{n}}^{0}\right) \in P_{i=1}^{n} \beta\left(X_{\alpha_{i}}\right)=\beta(X)$, so that this point has a neighborhood $V$ on which $\bar{f}(\cdot, y)$ varies by $<\epsilon$, for each $y$ in $Y$. But consider the corresponding neighborhood $V \times P_{\alpha \neq \alpha_{1}, \cdots, \alpha_{n}} \beta\left(X_{\alpha}\right)$ of $x^{0}$ in $P \beta\left(X_{\alpha}\right)$. It contains elements $\left(x^{1}, y^{1}\right)$ and $\left(x^{2}, y^{2}\right)$ of $X \times Y$ for which $a-\epsilon<f\left(x^{1}, y^{1}\right)$ $-f\left(x^{2}, y^{2}\right)$ so that

$$
\begin{aligned}
a-\epsilon & <\left|f\left(x^{1}, y^{1}\right)-f\left(x^{1}, y^{2}\right)\right|+\left|f\left(x^{1}, y^{2}\right)-f\left(x^{2}, y^{2}\right)\right| \\
& =\left|f\left(x^{1}, y^{1}\right)-f\left(x^{1}, y^{2}\right)\right|+\left|\bar{f}\left(x^{1}, y^{2}\right)-\bar{f}\left(x^{2}, y^{2}\right)\right|<2 \epsilon,
\end{aligned}
$$

and $3 \epsilon<a<3 \epsilon$, contradicting our assumption that $f$ has no continuous extension to $P \beta\left(X_{\alpha}\right)$, and completing the proof.

4. Some particular nontrivial Stone-Čech compactifications. The final portion of our proof of Theorem 1 reveals a class of Stone-Čech compactifications of a nontrivial, although certainly a very special, sort.

THEOREM 2. Let $\left\{X_{\alpha}\right\}$ be a set of uncountably many compact Hausdorff spaces, each having at least two points. For $b \in P X_{\alpha}$ let $X^{b}$ be the subspace of the 
product consisting of all points $x \in P X_{\alpha}$ with $x_{\alpha} \neq b_{\alpha}$ for at most countably many $\alpha$. Then $P X_{\alpha}$ is the Stone-Cech compactification of the proper subspace $X^{b}$.

The cardinality restrictions are only required to yield $X^{b}$ a proper subspace. Trivially $X^{b}$ is dense, and countably compact, since any sequence of points of $X^{b}$ has a cluster point (in the full product) which must, of course, lie in $X^{b}$. Just as in the proof of Theorem 1 we may assert that for $f \in C\left(X^{b}\right)$ and $\epsilon>0$ there are indices $\alpha_{1}, \cdots, \alpha_{n}$, for which $x, y \in X^{b}, x_{\alpha_{i}}=y_{\alpha_{i}}$, $i=1, \cdots, n$ imply $|f(x)-f(y)|<\epsilon$. For if not, we may proceed as in the previous proof (since we may alter countably many coordinates of a point in $X^{b}$ and remain in $X^{b}$ ) to sequences $\left\{x^{i}\right\}, \cdot\left\{y^{i}\right\}$ in $X^{b}$ and a sequence $\left\{F_{i}\right\}$ with just the properties which held there. But here $\left\{x^{i}\right\}$ has a cluster point in $X^{b}$, and any of its neighborhoods must contain $x^{i}$ and $y^{i}$ simultaneously for $i$ sufficiently large, so that we obtain the same contradiction.

Again, if $f$ does not have a continuous extension to $P X_{\alpha}$, we have an $x^{0}$ for which $\lim \sup _{x \rightarrow x^{0}} f(x)-\lim \inf _{x \rightarrow x_{0}} f(x)=a>0$. Taking $0<3 \epsilon<a$ and $\alpha_{1}, \cdots, \alpha_{n}$ the corresponding set of indices then, since we may write $X^{b}$ $=X \times Y$ with $X=P_{i=1}^{n} X_{\alpha_{i}}$, and $X^{b}$ is pseudo-compact, we arrive at the same final contradiction.

Some particular examples are intriguing. Take, for example, each $X_{\alpha}$ a compact topological group, $b$ the identity of the product group, so that $X^{b}$ and $\beta\left(X^{b}\right)$ are topological groups. Indeed take the simplest case with each $X_{\alpha}$ the two element group $\{0,1\}$, and let 0,1 be respectively the identity and $(1,1, \cdots)$. Extending the subgroup $X^{\mathbf{0}}$ of the full product to a maximal subgroup $Z$ not containing 1 , we have $Z$ and $1+Z$ complementary, since, for $x \notin Z, 1$ lies in the subgroup generated by $x$ and $Z$, or $1=x+z, z \in Z$, and $x=1+z \in 1+Z$. Thus in this case $Z$ and $\beta Z \backslash Z$ are homeomorphic, and all of the spaces $Z, \beta(Z) \backslash Z$ and $\beta(Z)$ are homogeneous (cf. [7]) ( ${ }^{7}$ ).

5. Products of pseudo-compact spaces. It is not true that a product of pseudo-compact spaces must be pseudo-compact. Indeed Novák [6] has recently given an example of a pair of countably compact completely regular spaces whose product is not even pseudo-compact $\left({ }^{8}\right)$. In this section we shall

(7) Numerous companions to Rudin's example of a homogeneous $X$ with $\beta(X) \backslash X$ not homogeneous can also be constructed via Theorem 2 . Let $K$ be a compact space, not totally disconnected, with an isolated point $k_{0}$ and cardinality $\boldsymbol{N}>\boldsymbol{\aleph}_{0}$. Let $Y$ be the product of $\boldsymbol{N}$ replicas of $K$, which, since $\aleph^{2}=\aleph$, contains the nonvoid subspace $X=\left\{y: y_{\alpha}=k\right.$ for $\aleph \alpha$ 's, for each $\left.k \in K\right\}$. Then $X$ is homogeneous since to take $x^{1}$ into $x^{2}$ only requires a permutation of the $\alpha$ 's. But $x \in X$ implies $Y^{x} \subset X$ and $x \in Y \backslash X$ implies $Y^{x} \subset Y \backslash X$ so that $\beta(X)=Y$ and $\beta(Y \backslash X)=Y$. Thus any homeomorphism of $\beta(X) \backslash X=Y \backslash X$ with itself extends to one of $\beta(Y \backslash X)=Y$ with itself. Consequently $\beta(X) \backslash X$ cannot be homogeneous since $\left(k_{0}, k_{0}, \cdots\right)$ is obviously its own component in $Y$, and $Y$ is not totally disconnected.

$\left.{ }^{8}\right)$ Novák's example was designed as a noncountably compact product of a pair of countably compact spaces. However, it contains an open and closed discrete infinite subspace, hence is clearly not pseudo-compact. A similar example has been given by Terasaka [9]. The writer is indebted to Dr. D. O. Ellis for reference to Novak's result. 
give some conditions which are sufficient to insure that a product be pseudocompact, and yield some analogous facts for other forms of compactness.

The generality of our results is increased somewhat by utilizing the notion of a $P$-point introduced by Gillman and Henriksen [2]. Such a point has the property that every countable intersection of its neighborhoods is a neighborhood (not necessarily open).

Theorem 3. Let $X$ and $Y$ be pseudo-compact. If $X$ is locally $\boldsymbol{\aleph}_{\gamma} \operatorname{compact}\left({ }^{9}\right)$, and each non-P-point of $Y$ has a base of neighborhoods of cardinality $\leqq \boldsymbol{\aleph}_{\gamma}$, then $X \times Y$ is pseudo-compact.

In particular this yields the fact that a product of two pseudo-compact spaces is pseudo-compact if one of the spaces is locally compact.

Proof. We shall show directly that $\beta(X \times Y)=\beta(X) \times \beta(Y)$, so that the conclusion will follow from Theorem 1 (unless either space is finite, in which case the conclusion is obvious). Let $f \in C(X \times Y)$. For $x_{0} \in X$, we have an $\boldsymbol{\aleph}_{\gamma}$ compact neighborhood $V$, and, as a consequence, we can assert that $\{f(x, \cdot)\}_{x \in V}$ is equicontinuous at any non- $P$-point $y_{0}$ in $Y$. Suppose not, so that for some $\epsilon>0, F_{W}=\left\{x: x \in V, \sup _{y \in W}\left|f(x, y)-f\left(x, y_{0}\right)\right| \geqq \epsilon\right\}$ is nonvoid for each neighborhood $W$ of $y_{0}$. Allowing $W$ to range over a base of neighborhoods $B$, of cardinality $\leqq \boldsymbol{N}_{\gamma},\left\{F_{W}\right\}$ forms a filter base on $V$ which has to have an adherent point $x_{1}$ in $V$. On the other hand, $\left(x_{1}, y_{0}\right)$ has a neighborhood $V_{1} \times W_{1}, W_{1} \in B$, on which $f$ varies by at most $\epsilon / 2$ so that $V_{1}$ cannot meet $F_{W_{1}}$, and $x_{1}$ cannot be adherent to $\left\{F_{W}\right\}$.

Since equicontinuity of any countable subset of $C(Y)$ is automatic at $P$ points, every countable subset of $\{f(x, \cdot)\}_{x \in V}$ is equicontinuous on $Y$. As we have noted, this implies that the full set is equicontinuous on $Y$, and by Ascoli's theorem it thus has compact closure in $C(Y)$. But this implies that the mapping $x \rightarrow f(x, \cdot)$ of $V$ into $C(Y)$ is continuous at $x_{0}$; for if the filter $\mathcal{F}$ on $V$ converges to $x_{0}$, then $\lim _{\mathcal{F}} f(x, y)=f\left(x_{0}, y\right)$ for $y \in Y$ and the image of the filter has at most the single adherent point $f\left(x_{0}, \cdot\right)$, hence must converge to $f\left(x_{0}, \cdot\right)$ by compactness. Since $V$ is a neighborhood of $x_{0}$, the mapping $x \rightarrow f(x, \cdot)$ of $X$ into $C(Y)$ is continuous at $x_{0}$, hence everywhere since $x_{0}$ was arbitrary, and, by Lemma $2, f$ has a continuous extension $\bar{f}$ to $X \times \beta(Y)$. Since $\beta(Y)$ is locally compact, the same argument (with $\beta(Y)$ taking the rôle of $X$ ) yields the desired extension of $\bar{f}$ to $\beta(X) \times \beta(Y)$, and the proof is complete.

Because of the basic asymmetry of the hypotheses on $X$ and $Y$ in Theorem 3 , its applicability is somewhat enhanced, as is easily seen by examples, by the trivial fact that a space which is a finite union of pseudo-compact subspaces is pseudo-compact (or the equally trivial fact that having a dense pseudo-compact subspace makes a space pseudo-compact, e.g. if $X$ is not locally $\boldsymbol{\aleph}_{\gamma}$ compact but contains a dense pseudo-compact subspace which is). One particular instance of the use of a finite decomposition in showing a

(9) That is, every point of $X$ has a neighborhood $V$ such that every open covering of $V$, of cardinality $\leqq \aleph_{1}$, has a finite subcovering. 
product $X \times Y$ is pseudo-compact should be mentioned, since it is a consequence of Theorem 3; we may disregard any open subset (of either space) which has compact closure. Specifically, if $X$ and $Y$ are pseudo-compact, and $W \subset Y$ is open and has compact closure, then $X \times W^{\prime}$ is pseudo-compact if and only if $X \times Y$ is pseudo-compact. For, as one easily sees from the topological conditions for pseudo-compactness, the closure of an open subset inherits this property. Thus if $X \times Y$ is pseudo-compact, $X \times W^{-\prime}$ inherits the property; since $X \times$ (boundary $W$ ) is pseudo-compact by Theorem 3 , the same is true of $X \times W^{\prime}$ as the union of these two sets. On the other hand, if $X \times W^{\prime}$ is pseudo-compact the same is true of $X \times Y$ as the union of this set and $X \times W^{-}$, which is pseudo-compact by Theorem $3\left({ }^{10}\right)$.

In showing an infinite product is pseudo-compact we need only consider countable partial products (cf. §2). This suggests use of the diagonal process which leads to the following, somewhat restricted, extensions of Theorem 3.

Theorem 4. Let $\left\{X_{\alpha}\right\}$ be a set of pseudo-compact spaces. Then $P X_{\alpha}$ is pseudo-compact in each of the following cases:

(a) all but one $X_{\alpha}$ is locally compact;

(b) for all but one $\alpha$, each non-P-point of $X_{\alpha}$ is a $G_{\delta}$; .

(c) for every $\alpha, X_{\alpha}$ is $\boldsymbol{\aleph}_{\gamma}$ compact and each non-P-point of $X_{\alpha}$ has a base of neighborhoods of cardinality $\leqq \boldsymbol{\aleph}_{\gamma}$.

Proof. (a) Since we need only consider, at worst, the countable case, let $X_{0}, X_{1}, \ldots$ be a sequence of spaces of the type described with $X_{0}$ the exceptional space. We shall show that any sequence $\left\{U^{n}\right\}$ of nonvoid canonical open neighborhoods in $P X_{j}$ has a cluster point.

Let $U^{n}=P U_{j}^{n}$, with $U_{j}^{n}=X_{j}$ for $j \geqq m_{n}$. Set $X_{j}^{*}=X_{j}$ for $j=0$ and all compact factors, and, for $X_{j}$ noncompact let $X_{j}^{*}$ be the one-point Alexandroff compactification of $X_{j}$, with $x_{j}^{*}$ the (nonisolated) point at infinity. Then $U_{j}^{n}$ remains open in $X_{j}^{*}$, and if we set $V_{j}^{n}=U_{j}^{n}$ for $j<m_{n}, V_{j}^{n}=X_{j}^{*}$ for $j \geqq m_{n}$, and $V^{n}=P V_{j}^{n}$, then $\left\{V^{n}\right\}$ forms a sequence of nonvoid open subsets of $P X_{j}^{*}$ $=X_{0} \times P_{j \geqq 1} X_{j}^{*}$, which is pseudo-compact by Tychonoff's theorem and Theorem 3. Moreover $U^{n}$ is dense in $V^{n}$ so that any cluster point of $\left\{V^{n}\right\}$ is also a cluster point of $\left\{U^{n}\right\}$. Thus if we can produce a cluster point of $\left\{V^{n}\right\}$ lying in the subspace $P X_{j}$ of $P X_{j}^{*}$ our proof will be complete.

Let $X_{j_{1}}$ be the first noncompact factor with $j \geqq 1$. $\left\{V_{j_{1}}^{n}\right\}$ has a cluster point in $X_{j_{1}}$, so that some closed neighborhood $W_{j_{1}}$ of $x_{j_{1}}^{*}$ does not meet some neighborhood of this cluster point, and, for infinitely many $n, V_{j_{1}}^{n} \cap W_{j_{1}}^{\prime} \neq \varnothing$. Let $\left\{n_{\imath}^{1}\right\}$ be the infinite sequence of integers $n$ for which this holds. Considering the next noncompact factor $X_{j_{2}}$ and $\left\{V_{j_{2}}^{n_{1}{ }^{1}}\right\}$ we can obtain in the same fashion a closed neighborhood $W_{j_{2}}$ of $x_{j_{2}}^{*}$ and a subsequence $\left\{n_{i}^{2}\right\}$ of $\left\{n_{1}^{1}\right\}$ for which $V_{j_{2}}^{n_{i}{ }^{2}} \cap W_{j_{2}}^{\prime} \neq \varnothing$. We continue; replacing $\left\{V^{n}\right\}$ by the diagonal subsequence (or the final subsequence, if the process terminates) and setting

$\left.{ }^{(10}\right)$ Indeed we only need to have $W^{-}$locally compact to conclude $X \times Y$ is pseudo-compact. 
$W_{j}=\varnothing$ for all other $j$ we have $V_{j}^{n} \cap W_{j}^{\prime} \neq \varnothing$ for all $n$ and $j$ with $n \geqq i$. Let $\tilde{V}_{j}^{n}=V_{j}^{n} \cap W_{j}^{\prime}$ for $j \leqq n$ and $=X_{j}^{*}$ otherwise; setting $\tilde{V}^{n}=P \tilde{V}_{j}^{n},\left\{\tilde{V}^{n}\right\}$ again forms a sequence of nonvoid open sets in $P X_{j}^{*}$, and as such has a cluster point $x$ in $P X_{j}^{*}$. But $x$ must also be a cluster point of $\left\{V^{n}\right\}$; for if $V=P V_{j}$ is a neighborhood of $x$ with $V_{j}=X_{j}^{*}$ for $j \geqq k$, then $\tilde{V}^{n} \cap V \neq \varnothing$ implies $V^{n} \cap V \neq \varnothing$ if $n$ exceeds $k$, since $\tilde{V}_{j}^{n} \subset V_{j}^{n}$ for $j \leqq n$. Moreover $x$ must lie in the subspace $P X_{j}$ of $P X_{j}^{*}$. For if $x_{k}=x_{\mathbf{k}}^{*}$ for some $k$ then the neighborhood $\left\{x^{\prime}: x_{k}^{\prime} \in W_{k}\right\}$ of $x$ in $P X_{j}^{*}$ meets infinitely many $\tilde{V}^{n}$ despite the fact that $W_{k} \cap \tilde{V}_{k}^{n}$ is void as soon as $n$ exceeds $k$. Thus $x$ is the required cluster point in $P X_{j}$ of $\left\{U^{n}\right\}$.

(b) We note first that if a point $y$ is a $G_{\delta}$ in a pseudo-compact space, then it has a countable base of neighborhoods. For by regularity: (i) $\{y\}$ $=\bigcap W_{j}$ with $W_{j}$ open and $W_{j+1}^{-} \subset W_{j}$; (ii) in order to show $\left\{W_{j}\right\}$ is a base of neighborhoods it suffices to show each closed neighborhood $W$ contains some $W_{j}$. But the collection $\left\{W_{j} \cap W^{\prime}\right\}$ is locally finite so that only finitely many elements of the collection are nonvoid; since $\cap\left(W_{j} \cap W^{\prime}\right)=\varnothing$ and the sets decrease some element of our sequence $\left\{W_{j} \cap W^{\prime}\right\}$ must be void.

Again we need only consider $X_{0}, X_{1}, \cdots$ with $X_{0}$ the exceptional space. Let $\left\{V^{n}\right\}$ be a sequence of canonical open neighborhoods in $P X_{j}$ with $U^{n}=P V_{j}^{n}$. Consider $\left\{V_{1}^{n}\right\}$. If possible, we select an $x_{1}$ in $X_{1}$ which lies in the closure of infinitely many of these sets, say those corresponding to $n_{1}^{1}, n_{2}^{1}, \ldots$; if not, the corresponding collection of open sets in $X_{1}$ cannot be locally finite so that for some $x_{1}$ each neighborhood of $x_{1}$ meets infinitely many of the sets while $x_{1}$ lies in the closure of only finitely many. Clearly $x_{1}$ cannot be a $P$ point, and thus has a countable base of neighborhoods; by the diagonal process we may then select a subsequence $\left\{n_{i}^{1}\right\}$ of the integers for which each neighborhood of $x_{1}$ meets almost all $V_{1}^{n_{i}{ }^{1}}$. Hence in either case we have an $x_{1}$ in $X_{1}$ and a subsequence $\left\{n_{t}^{1}\right\}$ with this property.

Considering $\left\{V_{2}^{n_{i}{ }^{1}}\right\}$, we then select in the same fashion an $x_{2}$ in $X_{2}$ and a subsequence $\left\{n_{i}^{2}\right\}$ of $\left\{n_{i}^{1}\right\}$ for which each neighborhood of $x_{2}$ meets almost all $V_{2}^{n_{i}{ }^{2}}$. Continuing, we obtain a point $\left(x_{1}, x_{2}, \cdots\right)$ of $P_{j z 1} X_{j}$ and a subsequence $\left\{V^{n_{i}}\right\}$ of our original sequence for which each neighborhood of $\left(x_{1}, x_{2}, \cdots\right)$ in $P_{j \geq 1} X_{j}$ meets almost all of the sets $\tilde{V}^{n_{i}^{i}}=\boldsymbol{P}_{j \geq 1} V_{j}^{n_{i}^{i}}$. Hence taking $x_{0}$ to be any cluster point in $X_{0}$ of $\left\{V_{0}^{n_{i}^{i}}\right\}$ yields the desired cluster point $\left(x_{0}, x_{1}, x_{2}, \cdots\right)$ of $\left\{V^{n}\right\}$.

(c) Here any countable product is actually countably compact. Let $\left\{x^{n}\right\}$ be any sequence of points in $P X_{j}$; for notational convenience let us write the sequence of integers as $\left\{n_{i}^{0}\right\}$. Let $j_{1}$ be the first $j$, if there are any, for which sequence of $j$ th coordinates $\left\{x_{j}^{n}\right\}$ has a cluster point $x_{j}$ in $X_{j}$ which is also a $P$-point. Then evidently $x_{j_{1}}$ occurs infinitely often in $\left\{x_{j_{1}}^{n}\right\}$, and we have a subsequence $\left\{n_{i}^{1}\right\}$ of the integers for which $x_{j_{1}}^{n_{1}{ }^{1}}=x_{j_{1}}$. If possible we select a least $j_{2}$ for which $\left\{x_{j_{2}}^{n_{1} 1}\right\}$ has a $P$-cluster point $x_{j_{2}}$, and thus obtain a subsequence $\left\{n_{1}^{2}\right\}$ of $\left\{n_{1}^{1}\right\}$ for which $x_{j_{2}}^{n_{i}^{2}}=x_{j_{2}}$, and continue. If the process termi- 
nates after $k(=0,1, \cdots)$ stages then $\left\{x^{n_{i}{ }^{k}}\right\}$ has the corresponding $j_{1}$ st, $\cdots$, $j_{k}$ th coordinates constant while, for each other $j,\left\{x_{j}^{n^{k}}\right\}$ can have only non- $P$ points as cluster points. If the process does not terminate, then for each $k \geqq 1, x_{j_{k}}^{n_{i} i}=x_{j_{k}}$ for almost all $i$ while, for every other $j,\left\{x_{j}^{n_{i} i}\right\}$ has only non- $P$ points as cluster points. Thus, in either case we may replace our sequence $\left\{x^{n}\right\}$ by a subsequence $\left\{y^{n}\right\}$ with the property that for each $i, y_{j_{i}}^{n}=x_{j_{i}}$ for almost all $n$ while, for everyother $j,\left\{y_{j}^{n}\right\}$ has only non- $P$-points as cluster points.

Now the points $x_{j_{1}}, x_{j_{2}}, \ldots$ are natural candidates for certain of the coordinates of a cluster point of $\left\{y^{n}\right\}$; we may select the remaining coordinates as follows. Let $F_{n}=\left\{y^{m}\right\}_{m \geqq n}, \mathcal{F}_{0}$ be the filter base $\left\{F_{n}\right\}$, and $p_{j}$ the projection of our product into $X_{j}$. Let $j_{1}^{*}$ be the first $j$, if there are any, not included in $\left\{j_{i}\right\}$, and select an adherent point $x_{j_{1}^{*}}^{*}$ in $X_{j_{1}}^{*}$ of $p_{j_{1}^{*}}^{*} \mathcal{F}_{0}$. Since $x_{j_{1}^{*}}^{*}$ is then a cluster point of $\left\{y_{j^{*}}^{n}\right\}$, we know it is not a $P$-point, hence has a base of neighborhoods, $B_{j_{1}}^{*}$, of cardinality $\leqq \boldsymbol{\aleph}_{\gamma}$. Thus the collection $\mathcal{F}_{1}=\{F \cap V$ : $\left.F \in \mathcal{F}_{0}, V=V_{j_{1}^{*}} \times \boldsymbol{P}_{j \neq j_{1}^{*}} X_{j}, V_{j_{1}^{*}} \in B_{j_{1}^{*}}\right\}$ forms a filter base of cardinality $\leqq \boldsymbol{\aleph}_{\gamma} \cdot \boldsymbol{\aleph}_{\gamma}$ $=\boldsymbol{\aleph}_{\gamma}$, so that $p_{j_{2}}^{*} \mathfrak{F}_{1}$ has an adherent point $x_{j_{2}}^{*}$ in $X_{j_{2}}^{*}$. Since $x_{j_{2}^{*}}^{*}$ is also adherent to the less fine filter base $p_{j_{2}}^{*} \mathcal{F}_{0}$, hence is a cluster point of $\left\{y_{j_{2}^{*}}^{n}\right\}, x_{j_{2}^{*}}$ cannot be a $P$-point. Again we obtain $B_{j_{2}}^{*}$ and form the filter base $\mathcal{F}_{2}=\left\{F \cap V: F \in \mathcal{F}_{1}, V\right.$ $\left.=V_{j_{2}}^{*} \times \boldsymbol{P}_{j \neq j_{2}}^{*} X_{j}, V_{j_{2}}^{*} \in B_{j_{2}}^{*}\right\}$ of cardinality $\leqq \boldsymbol{N}_{\gamma}$; clearly we may continue in this fashion to obtain filter bases $\mathcal{F}_{i}$ and non- $P$-points $x_{j_{i}^{*}}$ as long as any indices $j$ are not included among the $j_{i}$ and $j_{i}^{*}$. Whether this process terminates or not (or even starts) we obtain a point $\left(x_{1}, x_{2}, \cdots\right)$ in $P X_{j}$ which must be a cluster point of $\left\{y^{n}\right\}$. For any canonical neighborhood of this point contains one given by constraints of the form $y_{j_{i}} \in V_{j_{i}}, i=1, \cdots, k, y_{j_{i}^{*}}^{*} \in V_{j_{i}^{*}}$, $i=1, \cdots, m$, with $V_{j_{i}^{*}} \in B_{j_{i}^{*}}^{*}$ But almost all $y^{n}$ satisfy each of the constraints of the first set, hence almost all $y^{n}$ satisfy all the constraints of this set. On the other hand, infinitely many $y^{n}$ satisfy all constraints of the second set since, for each $F \in \mathcal{F}_{0}, F \cap\left\{y: y_{j_{i}^{*}} \in V_{j_{i}^{*}}, i \leqq m\right\}$ is an element of the filter base $\mathfrak{F}_{j_{m}}^{*}$, hence nonvoid. Thus we have obtained our cluster point and the proof is complete.

REMARKs. Simple modifications of the proof of (a) yield the following facts: (1) if $P Y_{\alpha}$ is pseudo-compact and $X_{\alpha}$ is an open pseudo-compact subspace of $Y_{\alpha}$ then $P X_{\alpha}$ is pseudo-compact; (2) a product of no more than $\boldsymbol{\aleph}_{\gamma}$ spaces, each $\boldsymbol{\aleph}_{\gamma}$ compact and all but one locally compact, is $\boldsymbol{\aleph}_{\gamma}$ compact. (1) contains (a) and follows by allowing $X_{\alpha}^{\prime}$ to assume the role of $x_{\alpha}^{*}$; for (2) one requires the simple result that the product of an $\boldsymbol{\aleph}_{\gamma}$ compact space and a compact space is $\boldsymbol{N}_{\gamma}$ compact( $\left.{ }^{11}\right)$. Indeed take the indices to be the ordinals

(11) Let $X$ be compact, $Y \boldsymbol{\kappa}_{\gamma}$ compact, and $\mathcal{F}$ a filter base of closed subsets of $X \times Y$, of cardinality $\leqq \boldsymbol{\aleph}_{\gamma}$. Assuming no point is adherent to $\mathcal{F}$ we have, for each $y \in Y$ an $F \in \mathcal{F}$ for which $F \cap(X \times\{y\})=\varnothing$, since $X \times\{y\}$ is compact. By a standard compactness argument this continues to hold in a neighborhood of $y$. Thus $\left\{U_{F}\right\}_{F \in F}$, with $U_{F}=\{y: F \bigcap(X \times\{y\})=\varnothing\}$ forms an open covering of $Y$ of cardinality $\leqq \aleph_{\gamma}$; for a finite subcovering $U_{F_{1}}, \cdots, U_{F_{n}}$, we obviously have $F_{1} \cap F_{2} \cap \ldots \cap F_{n}=\varnothing$, the desired contradiction. 
$\leqq \omega_{\gamma}, X_{1}=X_{1}^{*}$ to be the exceptional space, and $X_{\alpha}^{*}$ to be again the Alexandroff compactification of $X_{\alpha}$ for $\alpha>1$, so that $P X_{\alpha}^{*}$ is $\boldsymbol{\aleph}_{\gamma}$ compact. Then given a filter base $\mathcal{F}_{1}$ on $P X_{\alpha}$ of cardinality $\leqq \boldsymbol{N}_{\gamma}$ one chooses similarly restricted filter bases $F_{\alpha}$ and neighborhoods $W_{\alpha}$ of $x_{\alpha}^{*}$ (taking $W_{1}=\varnothing$ ) inductively by requiring that $W_{\beta}$ avoid some neighborhood of some point in $X_{\beta}$ adherent to $p_{\beta}\left(U_{\alpha<\beta} \mathcal{F}_{\alpha}\right)$ (which is possible since $\bigcup_{\alpha<\beta} \mathcal{F}_{\alpha}$ has cardinality $\leqq \boldsymbol{\aleph}_{\gamma} \cdot \boldsymbol{\aleph}_{\gamma}=\boldsymbol{N}_{\gamma}$ ) and selecting $\mathcal{F}_{\beta}$ as the filter base generated by $\bigcup_{\alpha<\beta} \mathcal{F}_{\alpha}$ and the set $\left\{x: x_{\beta} \notin W_{\beta}\right\}$. Then $\mathcal{F}_{\omega_{\gamma}}$ has an adherent point in $P X_{\alpha}^{*}$ which obviously must lie in $P X_{\alpha}$, and is adherent to $\mathscr{F}_{1}$.

Similarly the proof of (b) shows that (3) any countable product $P_{j=0}^{\infty} X_{j}$ of countably compact spaces for which, for $j>0$, we have each non-P-point of $X_{j} a$ $G_{\delta}$, is countably compact. The argument here is simpler; starting with a sequence $\left\{x^{n}\right\}$ of elements of $P X_{j}$ we choose $x_{1}$ as any cluster point of $\left\{x_{1}^{n}\right\}$; if $x_{1}$ is a $P$-point it must occur infinitely often in $\left\{x_{1}^{n}\right\}$. Otherwise $x_{1}$ has a countable base of neighborhoods, but in either case we may clearly begin an application of the diagonal process as before. Without reference to $P$-points (3) has an analogue for $\gamma>0$, whose proofs follows the final portion of that of (c): (4) a product of no more than $\boldsymbol{\aleph}_{\gamma}$ spaces, each $\boldsymbol{\aleph}_{\gamma}$ compact and all but one having at each point a base of neighborhoods of cardinality $\leqq \boldsymbol{\aleph}_{\gamma}$, is $\boldsymbol{\aleph}_{\gamma}$ compact. Again we take the indices to be the ordinals $\leqq \omega_{\gamma}$ and let $X_{1}$ be the exceptional space; given a filter base $\mathcal{F}_{1}$ of cardinality $\leqq \boldsymbol{\aleph}_{\gamma}$ one chooses, for $\alpha>1$, similarly restricted filter bases $\mathcal{F}_{\alpha}$ and elements $x_{\alpha}$ of $X_{\alpha}$ inductively by selecting $x_{\beta}$ adherent to $p_{\beta}\left(\mathrm{U}_{\alpha<\beta} \mathcal{F}_{\alpha}\right)$ and taking $\mathcal{F}_{\beta}$ as the filter base formed by the sets $F \cap\left\{x^{\prime}: x_{\beta}^{\prime} \in V_{\beta}\right\}$ where $F \in \bigcup_{\alpha<\beta} F_{\alpha}$ and $V_{\beta}$ is taken from our base of neighborhoods at $x_{\beta}$. Choosing $x_{1}$ adherent to $p_{1} \mathfrak{F}_{\omega_{\gamma}}$, clearly $x=\left(x_{1}, x_{2}, \cdots\right)$ is adherent to $\mathfrak{F}_{\omega_{\gamma}}$, hence $\mathcal{F}_{1}$.

Finally it should be noted that the argument used in (a) can be applied in other situations where a substitute for Tychonoff's theorem is available. For example (c) can be strengthened by replacing " $X_{\alpha}$ is $\boldsymbol{\aleph}_{\gamma}$ compact" by " $X_{\alpha}$ is the union of $\boldsymbol{\aleph}_{\gamma}$ open sets each having $\boldsymbol{\aleph}_{\gamma}$ compact closure." For then each $X_{\alpha}$ is locally $\boldsymbol{\aleph}_{\gamma}$ compact so that we can introduce, for each non $\boldsymbol{\aleph}_{\gamma^{-}}$ compact factor $X_{\alpha}$, its one-point $\boldsymbol{\aleph}_{\gamma}$-compactification $X_{\alpha}^{*}$ in just the way one constructs the Alexandroff compactification (with " $\boldsymbol{\aleph}_{\gamma}$-compact" replacing "compact"). Since each point at infinity has a base of neighborhoods of cardinality $\leqq \boldsymbol{N}_{\gamma}$, (c) implies $\boldsymbol{P} X_{\alpha}^{*}$ is pseudo-compact. But now the remainder of the argument given for (a) applies.

6. A related question. Even if it fails to be true that every continuous function on the subspace $P X_{\alpha}$ of $P \beta\left(X_{\alpha}\right)$ has a continuous extension to $P \beta\left(X_{\alpha}\right)$, it still might be the case that this holds for some other dense subspace homeomorphic to $P X_{\alpha}$, so that the Stone-Čech compactification of $P X_{\alpha}$ might be obtained from the compact space $P \beta\left(X_{\alpha}\right)$ via some other imbedding of $P X_{\alpha}$ therein. Some light is shed on this question by Theorem 4(a).

Call a point $z$ inessential to the space $Z$ if every $f \in C(Z \backslash\{z\})$ has a con- 
tinuous extension to $Z$. Evidently any ideal point $z$ of $\beta(Z)$ is inessential to $\beta(Z)$ and $\beta(\beta(Z) \backslash\{z\})=\beta(Z)$. By a theorem of Cech [1] which asserts that a nonvoid closed $G_{\delta}$ in the set of ideal points of $\beta(X)$ has high cardinality, we may conclude that $\beta(Z) \backslash\{z\}$ is then pseudo-compact; consequently any point $x$ of $P \beta\left(X_{\alpha}\right) \backslash P X_{\alpha}$ is inessential to $P \beta\left(X_{\alpha}\right)\left({ }^{12}\right)$. For letting $Z_{\alpha}=\beta X_{\alpha}$ if $x_{\alpha} \in X_{\alpha}, Z_{\alpha}=\beta X_{\alpha} \backslash\left\{x_{\alpha}\right\}$ if $x_{\alpha} \in X_{\alpha}$, we have $P Z_{\alpha}$ pseudo-compact by Theorem 4(a); thus $f \in C\left(P \beta\left(X_{\alpha}\right) \backslash\{x\}\right)$, being continuous on $P Z_{\alpha}$, has a continuous extension to $P \beta\left(Z_{\alpha}\right)=\boldsymbol{P} \beta\left(X_{\alpha}\right)$ by Theorem 1 , yielding the assertion.

Moreover any $z_{0}$ inessential to $\beta(Z)$ is inessential to $Z$. For suppose $f \in C\left(Z \backslash\left\{z_{0}\right\}\right)$ has no continuous extension to $Z$. We can of course take $f \geqq 1$. Let $\bar{f}$ be the extension of $f$ to $Z$ obtained by setting $\bar{f}\left(z_{0}\right)=1$, and let $G$ $=\left\{g: g \in C(\beta(Z)), 0 \leqq g \leqq 1, g\right.$ vanishes near $\left.z_{0}\right\}$ so that $\bar{f} g \in C(Z)$ for $g \in G$. Finally, let $F(z)=\sup _{G}(\bar{f} g)^{*}(z)$ for $z \in \beta(Z) \backslash\left\{z_{0}\right\}$. For $z_{1} \in \beta(Z) \backslash\left\{z_{0}\right\}$ we have disjoint neighborhoods $U$ of $z_{0}$ and $V$ of $z_{1}$ in $\beta(Z)$, and for any $g_{1} \in G$ which is 1 outside $U$ (such an element of $G$ exists by Urysohn's lemma), we have $F(z)=\left(\bar{f} g_{1}\right)^{*}(z)$ for $z \in V$. Indeed if this is not the case, for some $g_{2}$ in $G$ and $z$ in $V$ we have $\left(\bar{f} g_{2}\right)^{*}(z)>\left(\bar{f} g_{1}\right)^{*}(z)$; but some $g$ in $G$ satisfies $g g_{i}=g_{i}, i=1,2$, again by Urysohn's lemma, so that $(\bar{f} g)^{*}(z) g_{2}(z)=\left(\bar{f} g g_{2}\right)^{*}(z)=\left(\bar{f} g_{2}\right)^{*}(z)$ $>\left(\bar{f} g_{1}\right) *(z)=(\bar{f} g)^{*}(z) g_{1}(z)$, and $g_{2}(z)>g_{1}(z)=1$, a contradiction. Thus we see that $F \in C\left(\beta(Z) \backslash\left\{z_{0}\right\}\right)$; but $F$ has no continuous extension to $\beta(Z)$ since clearly $F \mid\left(Z \backslash\left\{z_{0}\right\}\right)=f$.

Suppose then that $Z$ is a dense subspace of $P \beta\left(X_{\alpha}\right)$ with the property that all continuous functions on $Z$ extend continuously to $P \beta\left(X_{\alpha}\right)$, i.e., with $\beta(Z)=P \beta\left(X_{\alpha}\right)$. Each point $x$ of $Z \backslash P X_{\alpha}$ is inessential to $\beta(Z)=P \beta\left(X_{\alpha}\right)$ as an element of $P \beta\left(X_{\alpha}\right) \backslash P X_{\alpha}$, and thus is inessential to $Z$. If we now assume that the inessential points of $Z$ form a discrete subspace, so does $Z \backslash P X_{\alpha}$. Since it is now simple to extend $f \in C\left(P X_{\alpha}\right)$ continuously to all of $Z\left({ }^{13}\right)$, we may further extend it to $\beta(Z)=P \beta\left(X_{\alpha}\right)$, so that $\beta\left(P X_{\alpha}\right)=P \beta\left(X_{\alpha}\right)$. Thus if the inessential points of $P X_{\alpha}$ form a discrete subspace, the existence of any imbedding of this space as a dense subspace of $P \beta\left(X_{\alpha}\right)$ which makes all elements of $C\left(P X_{\alpha}\right)$ continuously extendable yields the same property for the natural imbedding.

Added in proof (January 26, 1959). The reader may have noted that the example $X^{0}$ of $\S 4$ is a noncompact topological group on which all bounded continuous functions are almost periodic (being, essentially, continuous functions on the compact group $\beta\left(X^{0}\right)$ ). It is easily seen that any topological

(12) Of course every point of $\boldsymbol{P} \beta\left(X_{\alpha}\right)$ is inessential to this space if $\left\{\beta\left(X_{\alpha}\right)\right\}$ satisfies the cardinality requirements of Theorem 2 .

${ }^{(13)}$ To assign the appropriate value for this extension at $z \in Z \backslash P X_{\alpha}$ choose a $g \in C\left(P \beta\left(X_{\alpha}\right)\right)$ which is 1 near $z$ and vanishes on the remainder $R$ of $Z \backslash P X_{\alpha}$, and extend $f g$ to $Z \backslash\{z\}$ by assigning the value 0 to elements of $R$. This extension then lies in $C(Z \backslash\{z\})$ since $\left\{z^{\prime}:\left|g\left(z^{\prime}\right)\right|<\epsilon\right\}$ is a neighborhood of $R$, and thus has a further continuous extension to $Z$ since $z$ is inessential; the value at $z$ of this last function obviously meets our requirements. 
group $G$ for which $C(G)=$ A.P. $(G)$ (= real almost periodic functions) must be pseudo-compact; while the converse question must be left open we can note that if $G \times G$ is pseudo-compact then $C(G)=$ A.P. $(G)$, and $C(G \times G)$ $=$ A.P. $(G \times G)$. For $f \in C(G)$ implies $F:(x, y) \rightarrow f(x y)$ is an element of $C(G \times G)$ so that, by Lemma 1 , the left translates of $f$ are equicontinuous, and by Ascoli's theorem $f$ is almost periodic. Since we may view $\beta(G)$ and the Bohr compactification of $G$ as completions of $G$ under the natural uniform structures provided by $C(G)$ and A.P.( $G)$ respectively, these coincide as spaces and we may regard $\beta(G)$ as a compact group of which $G$ is a (albeit not closed, topological) subgroup. Since we may thus view $\beta(G \times G)=\beta(G) \times \beta(G)$ as a compact group of which $G \times G$ is a subgroup, every $f \in C(G \times G)$ is also almost periodic. Consequently $C(G \times G)=$ A.P. $(G \times G)$ iff $G \times G$ is pseudo-compact.

\section{REFERENCES}

1. E. Cech, On bicompact spaces, Ann. of Math. (2) vol. 38 (1937) pp. 823-844.

2. L. Gillman and M. Henriksen, Concerning rings of continuous functions, Trans. Amer. Math. Soc. vol. 77 (1954) pp. 340-362.

3. I. Glicksberg, The representation of functionals by integrals, Duke Math. J. vol. 19 (1952) pp. 253-261.

4. M. Henriksen and J. R. Isbell, On the Stone-Cech compactification of a product of two spaces, Bull. Amer. Math. Soc. Abstract 63-2-332.

5. E. Hewitt, Rings of real valued continuous functions, I, Trans. Amer. Math. Soc. vol. 64 (1948) pp. 45-99.

6. J. Novák, On the cartesian product of two compact spaces, Fund. Math. vol. 40 (1953) pp. 106-112.

7. W. Rudin, Homogeneity problems in the theory of $C_{\text {ech }}$ compactifications, Duke Math. J. vol. 23 (1956) pp. 409-419.

8. M. H. Stone, Applications of the theory of Boolean rings to general topology, Trans. Amer. Math. Soc. vol. 41 (1937) pp. 375-481.

9. H. Terasaka, On the cartesian product of compact spaces, Osaka Math. J. vol. 4 (1) (1952) pp. 11-15.

10. A. Tychonoff, Ueber die topologische Erweiterungen von Räumen, Math. Ann. vol. 102 (1930) pp. 544-561.

University of Notre Dame, Notre DAME, Ind. 